OPEN ACCESS

Edited by:

Maurizio Acampa

Siena University Hospital, Italy

Reviewed by:

Xinyi Leng,

The Chinese University of

Hong Kong, China

Lina Palaiodimou,

University General Hospital

Attikon, Greece

*Correspondence:

Shanshan Diao

diaoshanshan1015@163.com

Hongru Zhao

zhaohongru_soochow@163.com

tThese authors have contributed equally to this work

Specialty section:

This article was submitted to

Stroke,

a section of the journa

Frontiers in Neurology

Received: 18 June 2020 Accepted: 02 September 2020 Published: 16 October 2020

Citation:

Qin Y, Qian X, Luo X, Li Y, Wang D,

Jiang J, Zhang $Q$, Liu M, Xiao J, Zhang Y, Diao S and Zhao H (2020)

Association Between Plasma

Lipoprotein-Associated

Phospholipase A2 and Plaque

Vulnerability in TIA Patients With

Unilateral Middle Cerebral Artery

Stenosis. Front. Neurol. 11:574036.

doi: 10.3389/fneur.2020.574036

\section{Association Between Plasma Lipoprotein-Associated Phospholipase A2 and Plaque Vulnerability in TIA Patients With Unilateral Middle Cerebral Artery Stenosis}

Yiren Qin ${ }^{1+}$, Xiaoyan Qian ${ }^{2 \dagger}$, Xue Luo ${ }^{3}$, Yuanfang $\mathrm{Li}^{3}$, Dapeng Wang ${ }^{1}$, Jianhua Jiang ${ }^{1}$, Quanquan Zhang ${ }^{1}$, Meirong Liu ${ }^{1}$, Junhua Xiao ${ }^{4}$, Yan Zhang ${ }^{2}$, Shanshan Diao ${ }^{1 *}$ and Hongru Zhao ${ }^{1 *}$

${ }^{1}$ Department of Neurology, The First Affiliated Hospital of Soochow University, Suzhou, China, ${ }^{2}$ Department of Neurology, The First People's Hospital of Kunshan, Kunshan, China, ${ }^{3}$ Department of Neurology, Shiqian County People's Hospital, Tongren, China, ${ }^{4}$ Department of Gastroenterology, The First Affiliated Hospital of Soochow University, Suzhou, China

Background: Plasma lipoprotein-associated phospholipase A2 (Lp-PLA2) has emerged as a novel biomarker for coronary atherosclerosis. However, the association between Lp-PLA2 and plaque vulnerability in atherosclerosis of cervicocerebral arteries remains poorly defined, especially for intracranial atherosclerotic stenosis (ICAS). We aimed to investigate the association between Lp-PLA2 and plaque vulnerability in transient ischemic attack (TIA) patients with unilateral middle cerebral artery stenoses (MCAs).

Methods: In this study, a total of 207 patients were enrolled from April 2017 to April 2020. Clinical data were collected, and MCA plaques were examined with high-resolution magnetic resonance imaging (HRMRI). Baseline characteristics of patients were collected during hospitalization. Statistical comparisons were performed using Pearson's chi-squared test, Mann-Whitney U test, and the Breslow-Day/Tarone's test for the determination of heterogeneity in different age strata. Multivariate binary logistic analysis was used to investigate the potential independent predictors that were highly correlated to plaque vulnerability.

Results: The results showed that a high Lp-PLA2 level (>221 ng/ml) was associated with plaque vulnerability in TIA patients with unilateral MCAs. High Lp-PLA2 was independently associated with plaque vulnerability in patients $\leq 60$ years old [multivariate adjusted odds ratio $(\mathrm{OR})=9.854 ; 95 \% \mathrm{Cl}, 2.458-39.501]$ but not in patients $>60$ years old (multivariate adjusted $\mathrm{OR}=1.901 ; 95 \% \mathrm{Cl}, 0.640-5.650$ ). Predictors of plaque vulnerability in different age strata were also different.

Conclusion: Lp-PLA2 levels may be correlated to plaque vulnerability in TIA patients with unilateral MCAs and might be a diagnostic biomarker for plaque vulnerability in this kind of patients, especially for ones aged $\leq 60$ years old.

Keywords: Lp-PLA2, transient ischemic attack, middle cerebral artery, ICAs, HRMRI 


\section{INTRODUCTION}

Atherosclerosis (AS) is a kind of chronic inflammatory disease developed by heterogeneous causes (1). It plays an important role in the pathogenesis of the ischemic cerebrovascular disease. Plasma lipoprotein-associated phospholipase A2 (Lp-PLA2) has emerged as a novel inflammatory biomarker, which is involved in the initiation and progression of AS (2). Therefore, it is widely used as an inflammation predictor of atherosclerotic plaque in recent years (3). Previous studies have suggested that high expression of Lp-PLA2 correlated with poorer clinical outcome (4), increased cerebral infarction (5), and severer stenosis (6). However, the association between Lp-PLA2 and plaque vulnerability in atherosclerosis of cervicocerebral arteries, especially in ICAS patients has not been widely explored. For the last few years, high-resolution magnetic resonance imaging (HRMRI) was applied as a crucial noninvasive technique for the clinical determination of ICAS. It can objectively exhibit intracranial arterial wall morphology and atherosclerosis plaque characteristics by obtaining morphological measurements for atherosclerosis plaque composition $(7,8)$. However, it is much more expensive compared with traditional techniques, and is difficult to be applied in primary hospitals (9). Therefore, we aimed to investigate the association between Lp-PLA2 and plaque vulnerability in transient ischemic attack (TIA) patients with middle cerebral artery stenoses (MCAs) and explored whether Lp-PLA2 could be a potential diagnostic biomarker for plaque vulnerability in intracranial atherosclerotic artery.

\section{MATERIALS AND METHODS}

\section{Study Population}

This double-center study was reviewed and approved by the Ethics Committees of The First Affiliated Hospital of Soochow University and the Ethics Committees of The First People's Hospital of Kunshan, respectively. It was performed as a retrospective analysis of prospectively collected data between April 2017 and April 2020. Two hundred seven TIA patients with unilateral MCAs were recruited into our study. The inclusion criteria were as follows: (1) aged from 18 to 90 years; (2) diagnosed of first-ever TIA; (3) diagnosis of TIA fulfilled the World Health Organization (WHO) criteria with a complete clinical remission within $24 \mathrm{~h}$, and the time-based diagnosis has been verified in diffusion-weighted MRI demonstrating no relevant ischemic lesions; and (4) with CT angiography (CTA) evidence of unilateral MCAs of $70 \%$ or more and referred for HRMRI. Exclusion criteria were as follows: (1) previous antiplatelet, anticoagulant, thrombolytic, and statins therapies; (2) previous cerebral infarction; (3) previous cranial bleeding or hemorrhage; (4) previously diagnosed with thrombocytopenia or tumor, severe renal, hepatic, heart dysfunction; (5) previous heart disease (e.g., atrial fibrillation, coronary artery disease); (6) internal carotid artery dissection or cerebral vasculitis; (7) occlusion of unilateral MCA or acute cerebral infarction; (8) bilateral MCA stenosis; (9) severe stenosis or vulnerable plaque in the carotid artery; and (10) recurrent TIA.

\section{Data Collection}

All patients received the National Institutes of Health Stroke Scale (NIHSS) assessment by a qualified neurologist according to the symptom. Patients were etiologically assessed with other hospital workups including transcranial cerebral Doppler (TCD), echocardiography, and Holter monitor. Baseline characteristics of patients that may be associated with stroke risk were collected during hospitalization. MCA-M1 plaques were examined with HRMRI to assess the vulnerability of the plaques. Seven lipid parameters included total cholesterol (TC), total triglycerides (TG), high-density lipoproteins (HDLs), low-density lipoproteins (LDLs), apolipoprotein A (APOA), apolipoprotein B (APOB), and lipoprotein a (LPa).

\section{Measurement of Lp-PLA2}

Whole venous blood was drawn in tubes containing ethylene diamine tetraacetic acid (EDTA). The blood sample was centrifuged and was separated and placed in microtubes. Samples for measurement of Lp-PLA2 mass were handled simultaneously by a professional technician using the human Lp-PLA2 enzyme ELISA kit (Hotgen, Beijing, China). All protocols, e.g., precoat of specific antibody, incubation with primary and biotin-conjugated antibodies to Lp-PLA2, and optical density measurements, were performed according to the manufacturer's instructions.

\section{High-Resolution MRI Protocol}

HRMRI was performed using a 3.0-T MRI scanner (Ingenia, Philips Healthcare, Best, the Netherlands) with dedicated eightchannel head and neck unite coil. A standardized MR protocol was performed including routine MRI images and threedimensional (3D) time-of-flight (TOF) magnetic resonance angiography (MRA). The MCA-M1 segment for HRMRI scanning was obtained by a neuroradiologist. The sequence parameters were as follows: First, pre- and post-contrast T1WI image scanning used a turbo spin echo (TSE) measurement of the black blood two-dimensional (2D); repetition time (TR)/echo time (TE), 500/8.8 ms; TSE factor, 12; band width (BW), 393.1 $\mathrm{MHz}$; and number of average (NEX), 1. Next, the high-resolution T2WI image scanning was acquired also using TSE sequence; parameters were as follows: TR/TE, 2,500/271 ms; TSE factor, 100; BW, 561.9 MHz; and NEX, 2. After HR-T2WI, the highresolution PDWI image scanning was acquired also using TSE sequence; parameters were as follows: TR/TE, 2,000/40 ms; TSE factor, 60; BW, 425.2 MHz; and NEX, 1. Both acquisitions were run with a field of view (FOV) of $13 \mathrm{~cm}$, matrix size of 256 , slice thickness of $0.5 \mathrm{~mm}$, and non-gap, pixel size was $0.5 \times 0.5 \times 0.5$. Scan direction was parallel or perpendicular to the M1 segment. Three minutes later, contrast agent (gadopentetate dimeglumine, Gd-DTPA, Magnevist; Bayer Schering Pharma, Berlin, Germany) was injected intravenously with a dose of $0.1 \mathrm{mmol} / \mathrm{kg}$ and flowrate of $2 \mathrm{ml} / \mathrm{s}$.

\section{Image Analysis}

All images of the MCA plaques were reviewed and analyzed on a workstation by two well-trained neuroradiologists (one $>10$ years' experience and one $>5$ years' experience) independently blinded to the patient's clinical details. The differences between 
two neuroradiologists were solved by a senior consensus. The distributions, numbers, and locations of plaques were evaluated. Lipid-rich core, hemorrhage in the plaque, fibrous cap rupture, and/or calcified nodules protruding into the lumen were evaluated as previously described $(10,11)$. In summary, based on the previous researches (12-14), plaque vulnerability was defined following the three major criteria: (1) intraplaque hemorrhage (IPH, >150\% intensity compared with the adjacent normal gray matter), (2) plaque enhancement, and (3) plaques with heterogeneous signal intensity. The illustrative HRMRI images were demonstrated in Figure 1.

\section{Statistical Analysis}

Statistical analysis was performed with the SPSS version 24.0 program. The sample size determination of our study was done by PASS 15.0 software. Direct studies for patients with unilateral MCAs were not available to support a comparison between the normal and high levels of Lp-PLA2. Based on the preliminary data of our study, we estimated the percentage of patients with plaque vulnerability at normal and high levels of Lp-PLA2 at $\sim 20$ and 40\%, respectively. Meanwhile, we assumed that the group allocation of the normal and high levels of Lp-PLA2 was 1. Thus, a sample size of 180 patients was calculated as the minimum number required to yield a power of $80 \%$ with a twosided significance level of $5 \%$. Data are presented as median (IQR) for continuous variables and counts (percentages) for categorical data. Comparisons of patient characteristics were performed with the Mann-Whitney U test or Pearson's $\chi^{2}$ test. Multivariate logical regression analysis was used to detect the potential predictors of plaque vulnerability. The homogeneity assessment among different layers of age was determined by Breslow-Day and Tarone's test. The definition of low/high LP-PLA2 and cutoff of LP-PLA2 were concluded by receiver operating characteristic (ROC) curve and area under curve (AUC). A value of $p<0.05$ (two-sided) was considered to be statistically significant.
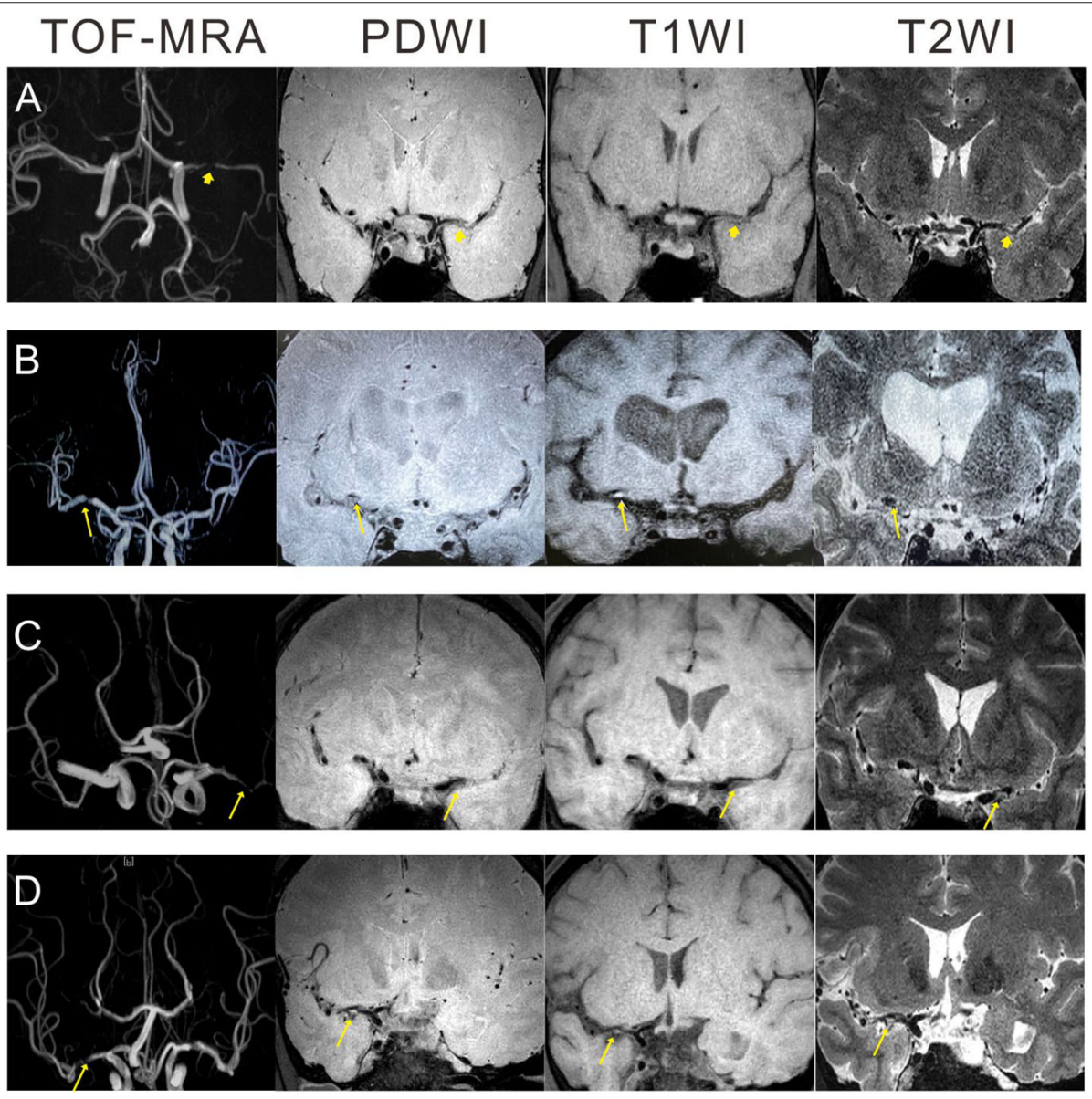

FIGURE 1 | Illustration of HRMRI images showing atherosclerotic plaques in patients with unilateral MCAs. (A) A 30 year-old man with non-vulnerable plaque: no high signal intensity on PDWI, T1WI, and T2WI. (B) A 70 year-old man with intraplaque hemorrhage: isointensity on PDWI, eccentric high signal intensity on T1WI, and hypointensity on T2WI. (C) A 42 year-old man with plaque enhancement: isointensity on PDWl, eccentric slight hyperintensity on T1WI, and slight hyperintensity on T2WI. (D) A 47 year-old man with heterogeneous signal intensity: mixed signal intensity on PDWI, T1WI, and T2WI. Arrowhead: non-vulnerable plaque; arrow: vulnerable plaque. HRMRI, high-resolution MRI; PDWI, proton density weighted imaging; TOF, time-of-flight; T2WI, T2-weighted imaging; T1WI, T1-weighted imaging; MCAs, middle cerebral artery stenosis. 


\section{RESULTS}

\section{Demographic Comparisons of the Studied Patients With Vulnerable and Non-vulnerable Plaque}

We enrolled 207 eligible TIA patients with unilateral MCAs who received HRMRI. Baseline characteristics of patients diagnosed with a vulnerable and non-vulnerable plaque are summarized in Table 1. The levels of HDL, APOB, and Lp-PLA2 were statistically different between patients with vulnerable and nonvulnerable plaque (Mann-Whitney test, $p$-values were 0.005 , $0.030,<0.001$, respectively). The percentage of smoke in patients with a vulnerable plaque was statistically higher than that with non-vulnerable plaque (57.1 vs. $39.4 \%, p=0.015)$. The illustrative HRMRI images for patients diagnosed with a vulnerable and non-vulnerable plaque are provided in Figure 1.

\section{Association of Lp-PLA2 With Plaque Vulnerability}

We next determined the sensitivity and specificity of Lp-PLA2 levels for the diagnosis of plaque vulnerability by using ROC analysis. The AUC of Lp-PLA2 levels for plaque vulnerability was 0.7455 (95\% CI, 0.6690-0.8219; $p<0.001$ ). The Lp-PLA2 level of $221 \mathrm{ng} / \mathrm{ml}$ was determined as the optimal cutoff value to predict plaque vulnerability. The sensitivity and specificity were 54.3 and $92.7 \%$, respectively (Figure 2). Thus, we classified LpPLA2 into a categorical variable (high Lp-PLA2 > $221 \mathrm{ng} / \mathrm{ml}$ ) and analyzed the association of high Lp-PLA2 with plaque vulnerability in different logistic models. In crude analysis, high Lp-PLA2 was associated with plaque vulnerability statistically (OR = 2.956; 95\% CI, 1.616-5.407). After age-sex, seven items of lipidemia, multivariate adjustments, and high Lp-PLA2 were still statistically related to the plaque vulnerability (Table 2 ).

\section{Effect of Lp-PLA2 on Plaque Vulnerability Among Different Age Strata}

To identify the optimal cutoff of age for high Lp-PLA2 to predict plaque vulnerability, age was stratified into a dichotomous variable, and homogeneity test for the effect of high Lp-PLA2 among different age strata was analyzed every 10 years. As shown in Table 3, the Breslow-Day/Tarone's test revealed a most statistically homogeneity in age $60\left(\chi^{2}=6.083\right.$ and $p=0.014$, and $\chi^{2}=6.070$ and $p=0.014$, respectively). We then performed a binary logistic analysis to investigate the potential interaction between age and high Lp-PLA2. The interaction of age and high Lp-PLA2 was statistically significant (OR $=4.720$; $95 \%$ CI, 1.358 $16.400, p=0.015)$. This indicated that the effect of high Lp-PLA2 on plaque vulnerability might be diminished with age.

\section{Predictors of Plaque Vulnerability Analyzed by Multivariate Binary Regression Model in Patients Aged $\leq 60$ or $>60$ Years}

We then performed binary logistic regression analyses in patients aged $\leq 60$ or $>60$ years. As summarized in Tables 4, 5, we found that high Lp-PLA2 was an important independent predictor for plaque vulnerability in patients $\leq 60$ years (Table $4, \mathrm{~B}=2.288$,

TABLE 1 | Characteristics of the studied patients with vulnerable and non-vulnerable plaque.

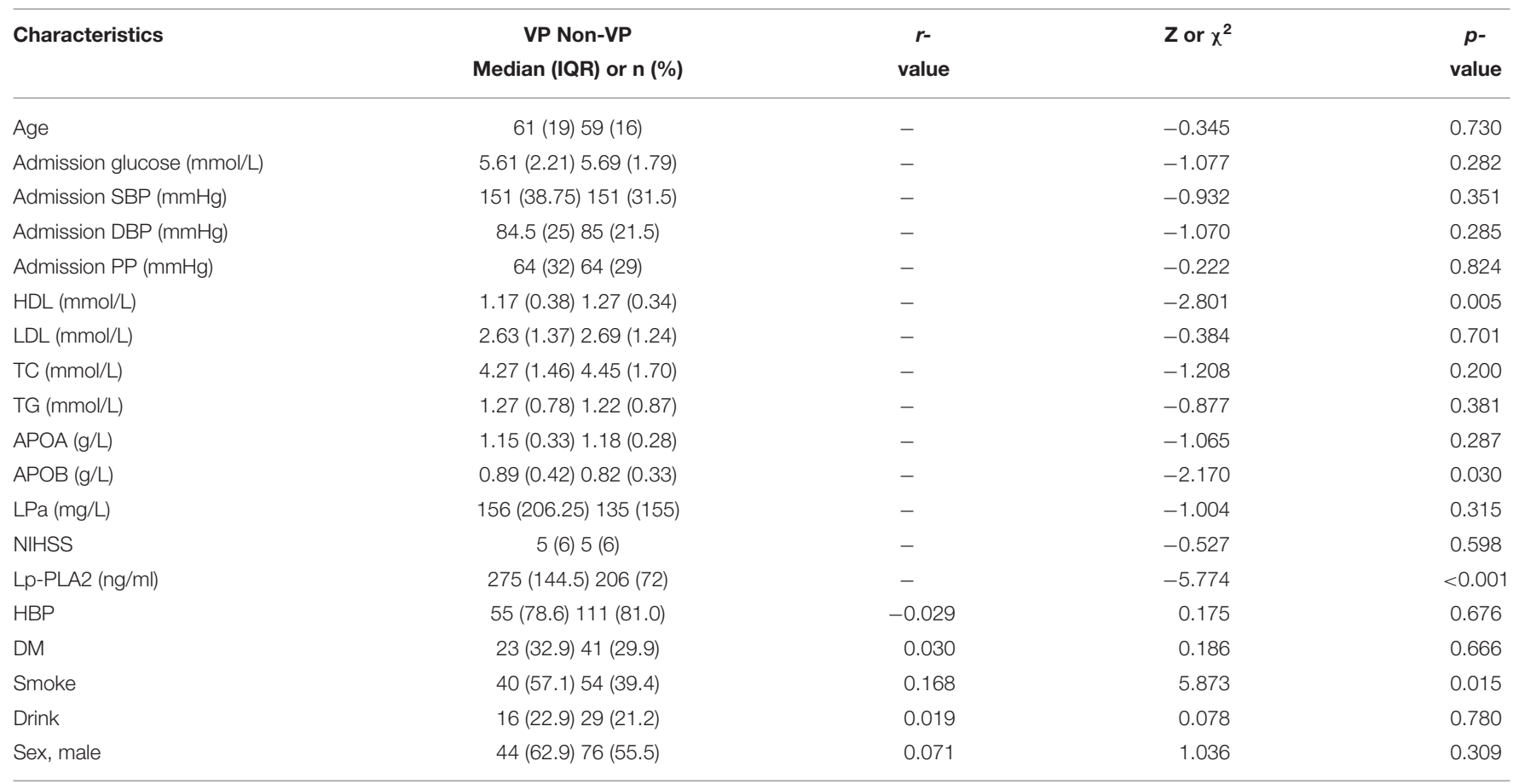

VP, vulnerable plaque; TC, total cholesterol; TG, total triglycerides; HDL, high-density lipoproteins; $L D L$, low-density lipoproteins; $A P O A$, apolipoprotein A; APOB, apolipoprotein B; LPa, lipoprotein a; NIHSS, the National Institutes of Health Stroke Scale; HBP, high blood pressure; DM, diabetes mellitus. 
$\mathrm{OR}=9.854,95 \% \mathrm{CI}, 2.458-39.501, p=0.001)$. However, in patients $>60$ years, this predictability achieved no statistical significance (Table 5; $\mathrm{B}=0.642$; OR $=1.901$; 95\% CI, 0.6405.650, $p=0.248$ ). In addition, HDL, APOB, and smoke were independent predictors of plaque vulnerability in patients $\leq 60$ years (Table 4; OR $=0.020,1.618,4.793$; 95\% CI, 0.001-0.441,

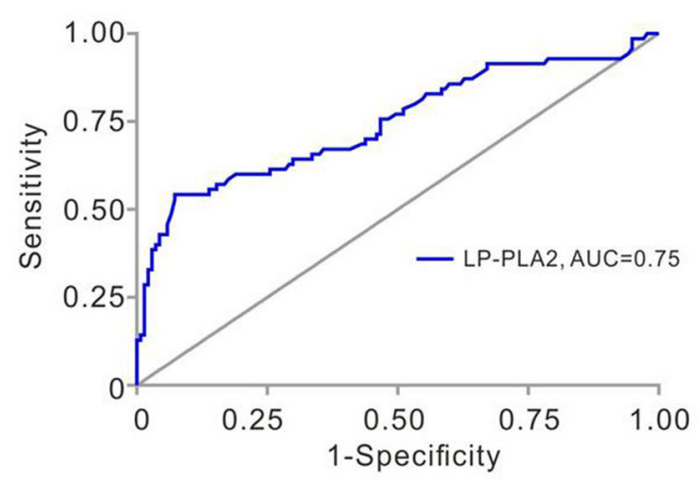

FIGURE 2 | Receiver operating characteristic (ROC) analysis of Lp-PLA2 in TIA patients with unilateral MCAs. Area under the ROC curve for Lp-PLA2 was 0.746 (95\% Cl, 0.669-0.822, $p<0.001)$. Lp-PLA2, lipoprotein-associated phospholipase A2; TIA, transient ischemic attack; MCAs, middle cerebral artery stenoses.

TABLE 2 | Odds ratios for diagnosis of plaque vulnerability in different models.

\begin{tabular}{lcc}
\hline & $\begin{array}{c}\text { High Lp-PLA2 } \\
\text { OR (95\% CI) }\end{array}$ & $\boldsymbol{p}$-value \\
\hline Crude & $2.956(1.616-5.407)$ & $<0.001$ \\
Age and sex & $2.859(1.551-5.269)$ & $<0.001$ \\
Lipidemia (seven) & $2.902(1.504-5.601)$ & 0.001 \\
Multivariate & $2.718(1.347-5.486)$ & 0.005 \\
\hline
\end{tabular}

$1.113-2.352,1.446-15.891$, respectively), and in patients $>60$ years, NIHSS and smoke were important predictors (Table 5; OR $=2.287$, 2.719; 95\% CI, 1.218-4.293, 1.196-6.179, respectively).

\section{ROC Analyses for Plaque Vulnerability in Patients Aged $\leq 60$ or $>60$ Years}

At last, we investigated the sensitivity and specificity of LpPLA2 levels for the diagnosis of plaque vulnerability in different age strata. In patients $\leq 60$ years, the AUC of Lp-PLA2 levels for plaque vulnerability was 0.8779 (95\% CI, 0.8023-0.9536, $p$ $<0.001)$. The Lp-PLA2 level of $221 \mathrm{ng} / \mathrm{ml}$ was determined as the optimal cutoff value to predict plaque vulnerability. The sensitivity and specificity were 70.6 and $97.5 \%$, respectively. However, there was no statistical predictive value of Lp-PLA2 [AUC, 0.5899 (95\% CI, 0.4645-0.7153), $p=0.1457$ ] in patients $>60$ years old with ROC analysis (Figure 3 ).

\section{DISCUSSION}

Intracranial atherosclerotic stenosis is one of the most common causes of cerebral infarction (CI) and TIA (15). Studies have shown that ICAS is predominant in Asian, African, and Hispanic origin, whereas extracranial arterial stenosis (ECAS) is more prevalent in Caucasians (16). In China, one-third case of ischemic stroke was related to ICAS, in which large intracranial arteries, e.g., MCA were involved (17). Previous studies have shown that about $10 \%$ of cases of TIA and $30-50 \%$ cases of CI were caused by MCA stenosis (18), and malignant MCA infarction is correlated with up to $80 \%$ mortality in the first week (19). Therefore, it is very important to evaluate MCA as accurately as possible (20). Conventionally, TCD, MRA, CTA, and digital substraction angiography (DSA) are performed to evaluate intracranial arterial stenosis. However, they cannot exhibit the wall morphology and atherosclerosis objectively. Although intracranial arterial stenosis is related to the occurrence of ischemic events (21), current studies have shown that the

TABLE 3 | Homogeneity determination of lipoprotein-associated phospholipase A2 (Lp-PLA2) across the age strata and interaction of Lp-PLA2 with age.

Chi-squared

Tests of homogeneity of the odds ratio

Breslow-Day

Tarone's

Variables

Interaction test of Lp-PLA2 with age

$\leq 60$

$(n=114)$

$>60$

$(n=93)$

Test for

interaction
6.083

6.070 p-value

0.014

0.014

VP, vulnerable plaque. 
TABLE 4 | Multivariate logistic regression analysis of variables associated with plaque vulnerability in studied patients aged $\leq 60$ years old.

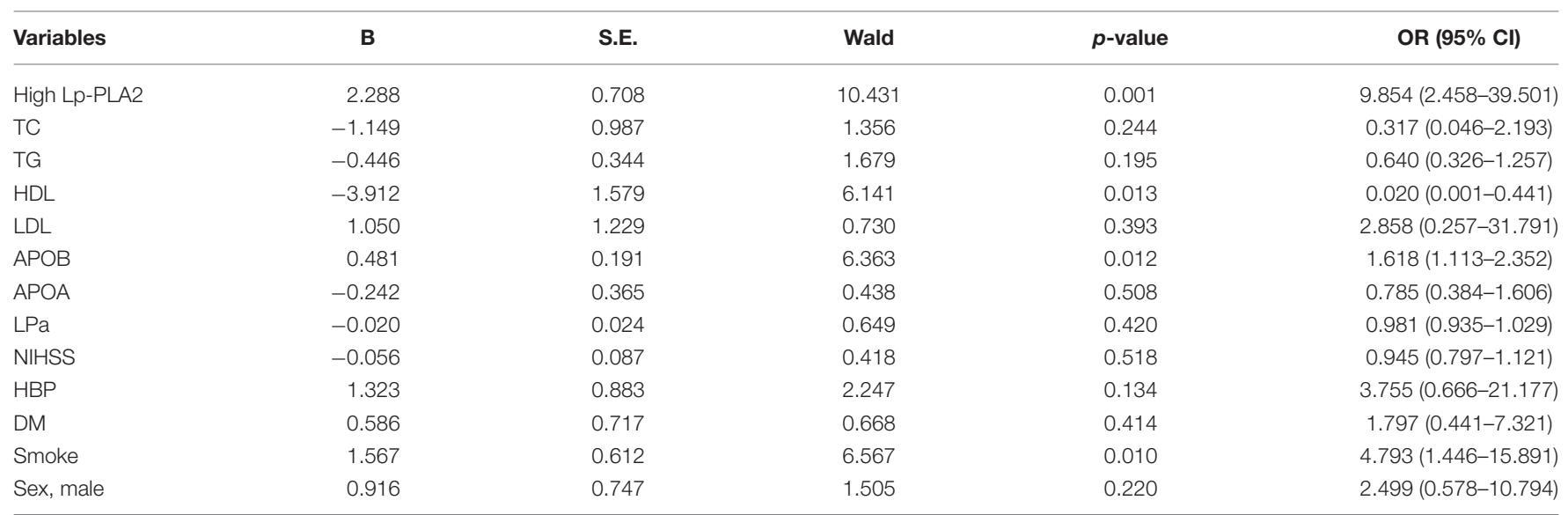

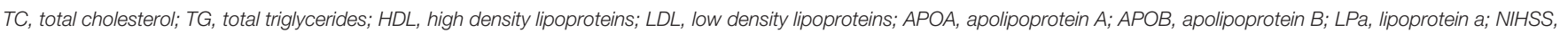
the National Institutes of Health Stroke Scale; HBP, high blood pressure; DM, diabetes mellitus.

TABLE 5 | Multivariate logistic regression analysis of variables associated with plaque vulnerability in studied patients aged > 60 years old.

\begin{tabular}{lccccr}
\hline Variables & B & S.E. & Wald & p-value & OR (95\% Cl) \\
\hline High Lp-PLA2 & 0.642 & 0.556 & 1.336 & 0.248 & $1.901(0.640-5.650)$ \\
TC & -0.029 & 0.097 & 0.091 & 0.763 & $0.971(0.803-1.174)$ \\
TG & -0.053 & 0.081 & 0.426 & 0.514 & 0.208 \\
HDL & -1.207 & 0.959 & 1.584 & 0.575 & $0.299(0.046-1.960)$ \\
LDL & -0.651 & 1.163 & 0.314 & 0.098 & $0.521(0.053-5.093)$ \\
APOB & 0.353 & 0.214 & 2.730 & 0.736 & $1.424(0.936-2.164)$ \\
APOA & 0.183 & 0.542 & 0.114 & 0.297 & $1.201(0.415-3.478)$ \\
LPa & 0.016 & 0.015 & 1.089 & 0.010 & $1.016(0.986-1.047)$ \\
NIHSS & 0.827 & 0.321 & 0.623 & 0.787 & $2.287(1.218-4.293)$ \\
HBP & 0.190 & 0.701 & 0.073 & 0.775 & $1.209(0.306-4.772)$ \\
DM & -0.193 & 0.674 & 0.082 & 0.017 & \\
Smoke & 1.000 & 0.419 & 5.703 & 0.423 &
\end{tabular}

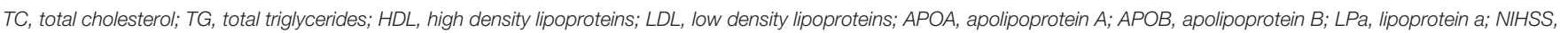
the National Institutes of Health Stroke Scale. HBP, high blood pressure; DM, diabetes mellitus.

occurrence of ischemic events in patients with symptomatic intracranial atherosclerosis is more relevant to the characteristics of a plaque rather than arterial stenosis by working with HRMRI (22). Therefore, it may be more important to evaluate plaque vulnerability in terms of predicting the risk of ischemic events rather than simply assess arterial stenosis (23).

Recently, HRMRI has been used as a promising modality to depict the intracranial artery wall and interpret plaque characteristics by combining a multitude of sequences. In the past few years, it has been hypothesized that the pathophysiology of intracranial atherosclerosis parallels that in the carotid artery by using HRMRI $(14,20,24)$. Previous histological comparison also pointed that the characteristics of plaque within the intracranial arteries were the same as those in carotid arteries, such as intraplaque hemorrhage, fibrous cap, and large lipid-rich necrotic core $(20,25)$. However, several current studies have shown slight discrepancies of intracranial plaque from those in carotid arteries (26). For example, fibrous cap is much thinner and is demonstrated with more ambiguous signal intensity than those in carotid plaques (27). Therefore, intracranial fibrous cap rupture may not be sensitively identified by HRMRI (28). With regard to intraplaque hemorrhage, the lower prevalence in MCA indicated that it is still far away from the "true" promising tool due to the smaller hemorrhagic volume and very complicated and muddled hemorrhagic components (29). Furthermore, some other limitations also influence its application in the evaluation of intracranial arterial plaques. HRMRI highly depends on its long acquisition time, coil quality, and resolution. Meanwhile, it is limited by its cost and availability, especially in primary hospitals (9). Therefore, it is of great significance to find a convenient, quick-applied, and relatively accurate technique to evaluate the plaque vulnerability of intracranial arteries as a supplementary diagnostic method.

AS is a chronic inflammatory disease. There are many inflammatory cytokines involved in the entire process of atheroma formation and development (30). Lp-PLA2 has 


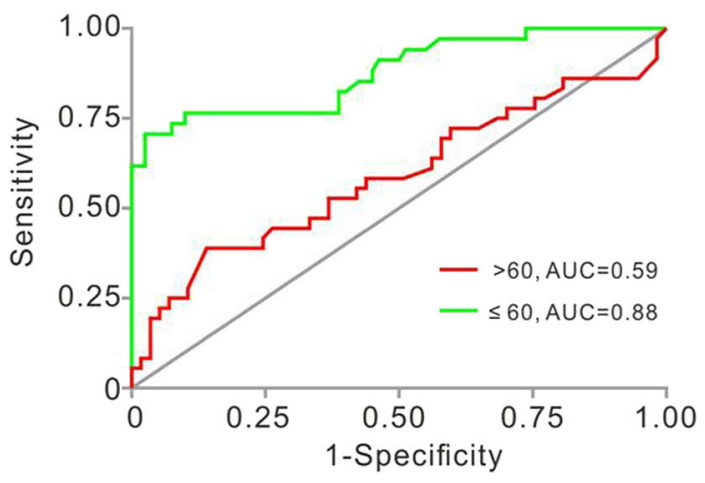

FIGURE 3 | Receiver operating characteristic (ROC) analyses of Lp-PLA2 in studied patients aged $\leq 60$ and $>60$ years old. AUC for Lp-PLA2 was 0.878 $(\leq 60,95 \% \mathrm{Cl}, 0.802-0.954, p<0.001)$ and $0.5899(>60,95 \% \mathrm{Cl}$ $0.4645-0.7153, p=0.1457)$. AUC, area under the ROC curve; Lp-PLA2, lipoprotein-associated phospholipase A2.

emerged as a novel biomarker of inflammation. Approximately $80 \%$ of Lp-PLA2 circulates bound to LDL, whereas the other $20 \%$ is bound to HDL and very low-density lipoprotein (VLDL) (31). Lp-PLA2 can hydrolyze oxPL at the surface of LDL, which produces a variety of cytokines, adhesion factors, and promotes the formation of atherosclerotic plaques (32). Meanwhile, histopathological examinations have shown that LP-PLA2 is highly expressed in the late necrotic core of atheromatous plaque, suggesting that it is involved in the formation and development of vulnerable atherosclerotic plaques (33). Theoretically, the determination of plasma Lp-PLA2 can predict the vulnerability of plaque. Nevertheless, previous studies were mostly focused on the correlation between carotid plaque vulnerability and Lp-PLA2 $(6,34)$. It is well-known that plaque vulnerability of carotid, especially in extracranial carotid, can be assessed by carotid ultrasound. In contrast, due to the presence of the skull, the intracranial artery can only be evaluated for the blood flow velocity with TCD and cannot be evaluated for plaque vulnerability directly (35). Thus, it is more valuable to investigate the correlation between Lp-PLA2 and plaque vulnerability in intracranial atherosclerotic arteries. In this study, our results suggested that the Lp-PLA2 level in patients with intracranial vulnerable plaque is statistically higher than that with nonvulnerable plaque, which is in accordance with the previous studies $(36,37)$. We also found that a high Lp-PLA2 level $(>221 \mathrm{ng} / \mathrm{ml}$ ) was associated with MCA plaque vulnerability in patients $\leq 60$ years but not in patients $>60$ years old. This may be because atherosclerosis is more prevalent in the elderly than in young adults. On the other hand, it may also be related to the decreased sensitivity of elderly patients to atherosclerotic inflammation (38).

Finally, some important limitations merit consideration: First, the sample size of studied patients was relatively small. Larger sample, prospective, multicenter studies or randomized trials of potent reversible pharmacological Lp-PLA2 inhibitors are further needed to clarify this issue in the future. Second, we only used qualitative approaches to discriminate plaque types. The visual evaluation was not absolutely objective, although the assessment was blinded to clinical information with senior consensus. Currently, quantitative measurements of signal intensity in MCAs offer standardization to distinguish the differences between vulnerable and non-vulnerable plaque by HRMRI (39). Until recently, MR signal features can be identified for the stenosis, plaque burden, and vulnerability (stenotic lumen area, stenotic wall area, plaque eccentricity, and plaque length), minimum luminal area, intraplaque hemorrhage, enhancement ratio, and dispersion of signal intensity (coefficient of variation) in a quantitative manner (40). Thus, this issue merits further quantitative validation. Third, the level of plasma Lp-PLA2 was assessed at a single time point due to its retrospective nature. Fourth, Lp-PLA2 activity tests were not performed in this study. However, Lp-PLA2 activity is highly associated with vascular inflammation and the vulnerability of atherosclerotic plaque (41). At last, we could not exclude other effects on plaque vulnerability, such as dietary habits and environmental effects.

In conclusion, Lp-PLA2 may be a promising biomarker to predict plaque vulnerability in TIA patients with unilateral MCAs, especially in patients $\leq 60$ years old. Testing of Lp-PLA2 as early as possible is necessary for patients with intracranial atherosclerotic stenosis.

\section{DATA AVAILABILITY STATEMENT}

The data that support the findings of this study are available from the corresponding author upon reasonable request.

\section{ETHICS STATEMENT}

The studies involving human participants were reviewed and approved by Ethics Committees of The First Affiliated Hospital of Soochow University AND the Ethics Committees of The First People's Hospital of Kunshan. The patients/participants provided their written informed consent to participate in this study.

\section{AUTHOR CONTRIBUTIONS}

HZ: study design. YQ and SD: study concepts. YQ and XQ: data acquisition. XQ and XL: statistical analysis. JJ: data supervisor. YL, DW, and QZ: manuscript preparation. YQ, ML, and JX: language editing. YZ, SD, and HZ: manuscript review. All authors contributed to the article and approved the submitted version.

\section{FUNDING}

This work was supported by the National Natural Science Foundation of China (grant number 81701051), Natural Science Foundation of Jiangsu Province (grant number BK20170362), and Natural Science Foundation of Jiangsu Province (grant number BK20170372). 


\section{REFERENCES}

1. Stoll G, Bendszus M. Inflammation and atherosclerosis: novel insights into plaque formation and destabilization. Stroke. (2006) 37:1923-32. doi: 10.1161/01.STR.0000226901.34927.10

2. Persson M, Berglund G, Nelson JJ, Hedblad B. Lp-PLA2 activity and mass are associated with increased incidence of ischemic stroke: a populationbased cohort study from Malmo, Sweden. Atherosclerosis. (2008) 200:1918. doi: 10.1016/j.atherosclerosis.2007.12.001

3. Ishida K, Cucchiara B. Therapeutic options to reduce Lp-PLA2 levels and the potential impact on vascular risk reduction. Curr Treat Options Cardiovasc Med. (2013) 15:313-21. doi: 10.1007/s11936-013-0239-4

4. Zhou F, Liu Y, Shi H, Huang Q, Zhou J. Relation between lipoproteinassociated phospholipase A2 mass and incident ischemic stroke severity. Neurol Sci. (2018) 39:1591-6. doi: 10.1007/s10072-018-3474-3

5. Kara H, Akinci M, Degirmenci S, Bayir A, Ak A, Nayman A, et al. High-sensitivity C-reactive protein, lipoprotein-related phospholipase A2, and acute ischemic stroke. Neuropsychiatr Dis Treat. (2014) 10:14517. doi: $10.2147 /$ NDT.S67665

6. Wang Y, Zhang J, Qian Y, Tang X, Ling $\mathrm{H}$, Chen $\mathrm{K}$, et al. Association of Lp-PLA2 mass and aysmptomatic intracranial and extracranial arterial stenosis in hypertension patients. PloS ONE. (2015) 10:e0130473. doi: 10.1371/journal.pone.0130473

7. Shin J, Chung JW, Park MS, Lee H, Cha J, Seo WK, et al. Outcomes after ischemic stroke caused by intracranial atherosclerosis vs dissection. Neurology. (2018) 91:e1751-9. doi: 10.1212/WNL.0000000000006459

8. Mandell DM, Mossa-Basha M, Qiao Y, Hess CP, Hui F, Matouk C, et al. Vessel wall imaging study group of the American society of N. Intracranial vessel wall MRI: principles and expert consensus recommendations of the American Society of Neuroradiology. Am J Neuroradiol. (2017) 38:21829. doi: 10.3174/ajnr.A4893

9. Bodle JD, Feldmann E, Swartz RH, Rumboldt Z, Brown T, Turan TN. High-resolution magnetic resonance imaging: an emerging tool for evaluating intracranial arterial disease. Stroke. (2013) 44:287-92. doi: 10.1161/STROKEAHA.112.664680

10. Sui B, Gao P, Lin Y, Jing L, Qin H. Distribution and features of middle cerebral artery atherosclerotic plaques in symptomatic patients: a $3.0 \mathrm{~T}$ high-resolution MRI study. Neurol Res. (2015) 37:391-6. doi: 10.1179/1743132815Y.0000000023

11. Cai JM, Hatsukami TS, Ferguson MS, Small R, Polissar NL, Yuan C. Classification of human carotid atherosclerotic lesions with in vivo multicontrast magnetic resonance imaging. Circulation. (2002) 106:136873. doi: 10.1161/01.CIR.0000028591.44554.F9

12. Kim JM, Jung KH, Sohn CH, Moon J, Han MH, Roh JK. Middle cerebral artery plaque and prediction of the infarction pattern. Arch Neurol. (2012) 69:1470-5. doi: 10.1001/archneurol.2012.1018

13. Yang WQ, Huang B, Liu XT, Liu HJ, Li PJ, Zhu WZ. Reproducibility of highresolution MRI for the middle cerebral artery plaque at 3T. Eur J Radiol. (2014) 83:e49-55. doi: 10.1016/j.ejrad.2013.10.003

14. Kim JM, Jung $\mathrm{KH}$, Sohn $\mathrm{CH}$, Moon J, Shin JH, Park J, et al. Intracranial plaque enhancement from high resolution vessel wall magnetic resonance imaging predicts stroke recurrence. Int J Stroke. (2016) 11:1719. doi: 10.1177/1747493015609775

15. Holmstedt CA, Turan TN, Chimowitz MI. Atherosclerotic intracranial arterial stenosis: risk factors, diagnosis, and treatment. Lancet Neurol. (2013) 12:1106-14. doi: 10.1016/S1474-4422(13)70195-9

16. Saber H, Amiri A, Thrift AG, Stranges S, Bavarsad Shahripour R, Farzadfard MT, et al. Epidemiology of intracranial and extracranial large artery stenosis in a population-based study of stroke in the Middle East. Neuroepidemiology. (2017) 48:188-92. doi: 10.1159/000479519

17. Qureshi AI, Caplan LR. Intracranial atherosclerosis. Lancet. (2014) 383:98498. doi: 10.1016/S0140-6736(13)61088-0

18. Qureshi AI, Feldmann E, Gomez CR, Johnston SC, Kasner SE, Quick DC, et al. Intracranial atherosclerotic disease: an update. Ann Neurol. (2009) 66:730-8. doi: 10.1002/ana.21768

19. Mohan Rajwani K, Crocker M, Moynihan B. Decompressive craniectomy for the treatment of malignant middle cerebral artery infarction. Br J Neurosurg. (2017) 31:401-9. doi: 10.1080/02688697.2017.1329518
20. Degnan AJ, Gallagher G, Teng Z, Lu J, Liu Q, Gillard JH. MR angiography and imaging for the evaluation of middle cerebral artery atherosclerotic disease. Am J Neuroradiol. (2012) 33:1427-35. doi: 10.3174/ajnr.A2697

21. Zhang DF, Chen YC, Chen H, Zhang WD, Sun J, Mao CN, et al. A high-resolution MRI study of relationship between remodeling patterns and ischemic stroke in patients with atherosclerotic middle cerebral artery stenosis. Front Aging Neurosci. (2017) 9:140. doi: 10.3389/fnagi.2017.00140

22. Xu WH, Li ML, Gao S, Ni J, Zhou LX, Yao M, et al. Plaque distribution of stenotic middle cerebral artery and its clinical relevance. Stroke. (2011) 42:2957-9. doi: 10.1161/STROKEAHA.111.618132

23. Chung GH, Kwak HS, Hwang SB, Jin GY. High resolution MR imaging in patients with symptomatic middle cerebral artery stenosis. Eur J Radiol. (2012) 81:4069-74. doi: 10.1016/j.ejrad.2012.07.001

24. de Havenon A, Mossa-Basha M, Shah L, Kim SE, Park M, Parker D, et al. Highresolution vessel wall MRI for the evaluation of intracranial atherosclerotic disease. Neuroradiology. (2017) 59:1193-202. doi: 10.1007/s00234-017-1925-9

25. Chen XY, Wong KS, Lam WW, Zhao HL, Ng HK. Middle cerebral artery atherosclerosis: histological comparison between plaques associated with and not associated with infarct in a postmortem study. Cerebrovasc Dis. (2008) 25:74-80. doi: 10.1159/000111525

26. Yang WJ, Wong KS, Chen XY. Intracranial atherosclerosis: from microscopy to high-resolution magnetic resonance imaging. J Stroke. (2017) 19:24960. doi: 10.5853 /jos.2016.01956

27. Harteveld AA, Denswil NP, Siero JC, Zwanenburg JJ, Vink A, Pouran B, et al. Quantitative Intracranial atherosclerotic plaque characterization at 7T MRI: an ex vivo study with histologic validation. Am J Neuroradiol. (2016) 37:802-10. doi: 10.3174/ajnr.A4628

28. $\mathrm{Xu} \mathrm{W}$. High-resolution MRI of intracranial large artery diseases: how to use it in clinical practice? Stroke Vasc Neurol. (2019) 4:102-4. doi: 10.1136/svn-2018-000210

29. Xu WH, Li ML, Gao S, Ni J, Yao M, Zhou LX, et al. Middle cerebral artery intraplaque hemorrhage: prevalence and clinical relevance. Ann Neurol. (2012) 71:195-8. doi: 10.1002/ana.22626

30. Spence JD, Solo K. Resistant atherosclerosis: the need for monitoring of plaque burden. Stroke. (2017) 48:1624-9. doi: 10.1161/STROKEAHA.117.017392

31. Caslake MJ, Packard CJ, Suckling KE, Holmes SD, Chamberlain P, Macphee $\mathrm{CH}$. Lipoprotein-associated phospholipase $\mathrm{A}(2)$, platelet-activating factor acetylhydrolase: a potential new risk factor for coronary artery disease. Atherosclerosis. (2000) 150:413-9. doi: 10.1016/S0021-9150(99)00406-2

32. Huang F, Wang K, Shen J. Lipoprotein-associated phospholipase A2: the story continues. Med Res Rev. (2020) 40:79-134. doi: 10.1002/med.21597

33. Otsuka F, Zhao X, Trout HH, Qiao Y, Wasserman BA, Nakano $\mathrm{M}$, et al. Community-based statins and advanced carotid plaque: role of CD163 positive macrophages in lipoprotein-associated phospholipase A2 activity in atherosclerotic plaque. Atherosclerosis. (2017) 267:78-89. doi: 10.1016/j.atherosclerosis.2017.10.014

34. Jackisch L, Kumsaiyai W, Moore JD, Al-Daghri N, Kyrou I, Barber TM, et al. Differential expression of Lp-PLA2 in obesity and type 2 diabetes and the influence of lipids. Diabetologia. (2018) 61:115566. doi: 10.1007/s00125-018-4558-6

35. Sharma VK, Wong KS, Alexandrov AV. Transcranial doppler. Front Neurol Neurosci. (2016) 40:124-40. doi: 10.1159/000448309

36. Du Y, Wei F, Dong Z, Liu J, Jiang W, Lu Q. Prognostic value of serum LPPLA2 and hs-CRP in unstable atherosclerotic plaques. Clin Exp Hypertens. (2011) 33:113-6. doi: 10.3109/10641963.2010.531836

37. Chung JW, Oh MJ, Cho YH, Moon GJ, Kim GM, Chung CS, et al Distinct roles of endothelial dysfunction and inflammation in intracranial atherosclerotic stroke. Eur Neurol. (2017) 77:211-9. doi: 10.1159/000460816

38. Wu D, Meydani SN. Age-associated changes in immune and inflammatory responses: impact of vitamin E intervention. J Leukoc Biol. (2008) 84:90014. doi: $10.1189 / \mathrm{jlb} .0108023$

39. Meng Y, Li M, Yu Y, Xu Y, Gao S, Feng F, et al. Quantitative score of the vessel morphology in middle cerebral artery atherosclerosis. J Neurol Sci. (2019) 399:111-7. doi: 10.1016/j.jns.2019.02.025

40. Shi Z, Li J, Zhao M, Peng W, Meddings Z, Jiang T, et al. Quantitative histogram analysis on intracranial atherosclerotic plaques: a highresolution magnetic resonance imaging study. Stroke. (2020) 51:2161-9. doi: 10.1161/STROKEAHA.120.029062 
41. Bonnefont-Rousselot D. Lp-PLA2, a biomarker of vascular inflammation and vulnerability of atherosclerosis plaques. Ann Pharm Fr. (2016) 74:1907. doi: 10.1016/j.pharma.2015.09.002

Conflict of Interest: The authors declare that the research was conducted in the absence of any commercial or financial relationships that could be construed as a potential conflict of interest.
Copyright $\odot 2020$ Qin, Qian, Luo, Li, Wang, Jiang, Zhang, Liu, Xiao, Zhang, Diao and Zhao. This is an open-access article distributed under the terms of the Creative Commons Attribution License (CC BY). The use, distribution or reproduction in other forums is permitted, provided the original author(s) and the copyright owner(s) are credited and that the original publication in this journal is cited, in accordance with accepted academic practice. No use, distribution or reproduction is permitted which does not comply with these terms. 